\title{
Riscos e benefícios da terapia hormonal no climatério
}

\author{
Risks and benefits of hormonal therapy not climatério
}

Riesgos y beneficios de la terapia hormonal en el climateral

\begin{abstract}
Glauce Lenira Silva Belém ${ }^{1 *}$, Stefano dos Santos Adorno ${ }^{1}$, Dária Barroso Serrão das Neves ${ }^{1}$, Livia Laura do Santos Rocha1, Moisés Castro Saback¹.
\end{abstract}

\section{RESUMO}

Objetivo: Revisar os Riscos e benefícios da terapia hormonal no climatério. Métodos: Foi realizada uma revisão bibliográfica de caráter sistemático para a promoção de um estudo básico, exploratório e qualitativo fundamentado em artigos científicos. A coleta de dados deste estudo inclui, essencialmente, a pesquisa por artigos publicados entre os anos de 2006 a 2017 e indexados em meio às seguintes bases de dados: nos quais foram aplicados os descritores: Climatério, menopausa, TH, benefícios. Utilizando assim critérios de inclusão e exclusão. Resultados: Observou-se que os resultados encontrados, com base nos artigos/publicações que após a menopausa, em adição à perda da capacidade reprodutiva, os tecidos e órgãos da mulher perdem o suporte uma vez que é assegurado pelo estrogênio, sendo necessário repor os hormônios perdidos, principalmente afim de oferecer uma qualidade de vida nesta fase. Embora o uso da terapia hormonal (TH) seja rodeada de frequentes dúvidas ela tem sido considerada como um importante aliado no alívio dos sintomas e redução no aparecimento de doenças nesta fase da vida, não sendo isenta de riscos Conclusão: A terapia hormonal pós-menopausa é indicada tanto para fins terapêuticos quanto profiláticos, o que pode aumentar a longevidade feminina. $O$ tratamento deve ser individualizado para identificar o tipo de terapia hormonal mais apropriado.

Palavras-Chave:Menopausa, Terapia Hormonal, Saúde, Benefícios.

\begin{abstract}
Objective: To review the risks and benefits of hormonal therapy in climacteric. Methods: A systematic bibliographic review was carried out to promote a basic, exploratory and qualitative study based on scientific articles. The data collection of this study essentially includes the search for articles published between the years 2006 to 2017 and indexed through the following databases: in which the following descriptors were applied: Climacteric, menopause, TH, benefits. Using the inclusion and exclusion criteria. Results: It was observed that the results found, based on post-menopausal articles / publications, in addition to loss of reproductive capacity, women's tissues and organs lose their support since it is ensured by estrogen, and it is necessary to replace lost hormones, mainly in order to offer a quality of life at this stage. Although the use of hormone therapy (HT) is often fraught with doubts, it has been considered as an important ally in relieving symptoms and reducing the onset of diseases at this stage of life, without risk. Conclusion: Postmenopausal hormone therapy is indicated for both therapeutic and prophylactic purposes, which may increase female longevity. Treatment should be individualized to identify the most appropriate type of hormonal therapy.
\end{abstract}

Keywords: Menopause, Hormonal Therapy, Health, Benefits.

${ }_{1}$ Universidade Estadual do Amazonas (UEA), Manaus - AM. * E-mail:glaucebelem.med@gmail.com 


\section{RESUMEN}

Objetivo: Revisar los Riesgos y beneficios de la terapia hormonal en el climaterio. Métodos: Se realizó una revisión bibliográfica de carácter sistemático para la promoción de un estudio básico, exploratorio y cualitativo fundamentado en artículos científicos. La recolección de datos de este estudio incluye, esencialmente, la investigación por artículos publicados entre los años 2006 a 2017 e indizados en medio de las siguientes bases de datos: en los cuales se aplicaron los descriptores: Climaterio, menopausia, TH, beneficios. Resultados: Los resultados obtenidos, con base en los artículos / publicaciones que tras la menopausia, además de la pérdida de la capacidad reproductiva, los tejidos y órganos de la mujer pierden el soporte ya que es asegurado por el estrógeno, siendo necesario reponer las hormonas perdidas, principalmente a fin de ofrecer una calidad de vida en esta fase. Aunque el uso de la terapia hormonal (TH) está rodeado de frecuentes dudas, se ha considerado como un importante aliado en el alivio de los síntomas y reducción en la aparición de enfermedades en esta fase de la vida, no siendo exenta de riesgos. Conclusión: La terapia hormonal posmenopáusica es indicada tanto para fines terapéuticos como profilácticos, lo que puede aumentar la longevidad femenina. El tratamiento debe ser individualizado para identificar el tipo de terapia hormonal más apropiada.

Palabras clave: Menopausia, Terapia Hormonal, Salud, Beneficios.

\section{INTRODUÇÃO}

O climatério, termo de origem grega (klimakter), faz referência ao topo de uma escada, sugerindo ascensão a um diferente patamar o estágio da vida. $O$ termo menopausa (do grego mens = mês; pausis = pausa) referese à parada das menstruações (BISOGNIN, 2016).

Ainda que o climatério seja um fenômeno normal, pode ter consequências potencialmente nocivas, com repercussões sobre vários sistemas, além do urogenital. Em termos científicos, climatério é apenas um período de vida, entre os anos reprodutivos e a senilidade (BISOGNIN, 2016).

Características e intensidades dos sintomas do climatério variam com as atitudes culturais. Na maioria das sociedades ocidentais, em que a frequência de sintomas climatéricos é elevada, a ênfase está relacionada no típico físico das mulheres. Por outro lado, em muitas culturas não ocidentais, há aumento do status da mulher pós-menopáusica (RIBEIRO et al, 2013).

Após a menopausa, o epitélio do trato genital se torna mais fino, as secreções das glândulas sudoríparas e sebácea da vulva diminuem, as glândulas de Bartholin atrofiam, a lubrificação diminui e a vagina se torna mais estreita, com a consequente redução de suas rugas e elasticidade (PARDINI, 2014).

Normalmente as mulheres têm menopausa com média de idade de 51 anos, aponta-se que a grande maioria algo em torno de $95 \%$ se tornando menopausadas entre as idades de 45 a 55 anos. Durante a transição da menopausa e pós-menopausa, muitas mulheres experimentam ondas de calor moderadas a graves $O$ estrogênio sistêmico é o tratamento mais eficaz disponível para alívio de ondas de calor. As mulheres na pós-menopausa também podem apresentar sintomas vulvovaginais, incluindo dispareunia, que pode ser efetivamente tratada pela administração local de estrogênio (MANSON, 2013).

A terapia hormonal $(\mathrm{TH})$ da menopausa é considerada um tratamento eficaz para os sintomas da menopausa, principalmente os sintomas vasomotores (VMS), a síndrome urinária da menopausa (GSM) e para prevenir a perda óssea e fratura. Entretanto, para avaliar se a terapia é eficaz em qualquer grupo, os benefícios para a qualidade de vida precisam ser considerados juntamente com os riscos para a saúde e os recursos (custos) associados ao uso (VALENÇA; GERMANO, 2010).

Os riscos de TH diferem dependendo do tipo, dose, duração do uso, via de administração, tempo de iniciação e do progestogênio utilizado. O tratamento deve ser individualizado para identificar o tipo de TH mais apropriado, dose, formulação, via de administração e duração do uso, usando as melhores evidências

REAS/EJCH | Vol. 11 (4) | e244 | DOI: https://doi.org/10.25248/reas.e244.2019 Página 2 de 7 
disponíveis para maximizar os benefícios e minimizar as reavaliações periódicas dos benefícios e riscos de continuar ou interromper a TH. (WHITMER et al, 2011).

\section{MÉTODOS}

Foi realizada uma revisão bibliográfica de caráter sistemático para a promoção de um estudo básico, exploratório e qualitativo fundamentado em artigos científicos e demais produções científico-acadêmicas que se mostrem úteis e pertinentes à pesquisa em tela (FONSECA, 2012).

A coleta de dados deste estudo inclui, essencialmente, a pesquisa por artigos publicados entre os anos de 2006 a 2017 e indexados em meio às seguintes bases de dados:SciELO (Scientific Electronic Library Online), PubMed (US National Library of Medicine Nationallnstitutesof Health), ScienceDirect (Elsevier'sleadinginformationsolution) e BIREME (Biblioteca Regional de Medicina do Centro LatinoAmericano e do Caribe de Informação em Ciências da Saúde), nos quais foram aplicados os descritores: Climatério, menopausa, TH, benefícios. Utilizando assim critérios de inclusão e exclusão

\section{RESULTADOS E DISCUSSÃO}

Após a menopausa, em adição à perda da capacidade reprodutiva, tecidos e órgãos da mulher perdem o suporte antes assegurado pelo estrogênio, sendo necessário repor os hormônios perdidos, principalmente 0 estradiol, para harmonizar a perda dos hormônios femininos e oferecer uma melhor qualidade de vida nesta fase. Esta ação de prescrever terapia hormonal para a mulher exige arte e ciência. Arte, no desenho de cada prescrição, de modo específico, para cada mulher (prescrição individualizada, personalizada). Ciência, no conhecimento médico de riscos e benefícios do uso de estradiol nos anos pós-menopausa. (LOMÔNACO et al, 2015).

\section{Riscos e benefícios da terapia hormonal no climatério}

Embora uso da terapia hormonal (TH) ainda seja rodeada de dúvidas e incertezas, baseadas nas publicações sobre o seu uso, ela tem sido citada como um importante aliado no alívio dos sintomas e redução no aparecimento de doenças nesta fase da vida, não sendo totalmente isenta de riscos (BISOGNIN, 2016).

Evidências dos riscos para a saúde associados ao uso de TH vinham se acumulando em estudos como o Heart and Estrogen/progestinReplacementStudy (HERS), juntamente com os resultados do Women's Health Initiative (WHI) de 2002 e 2004, nos quais foram obtidos dados relacionando os resultados negativos ao início da terapia em paciente com idade avançada, via de administração, doses altas e tempo de menopausa, o que levou ao aparecimento do conceito de invidualização, passando-se a valorizar a idade da paciente, tempo de menopausa, sintomas, dose, vias de administração, comorbidades, entre outros, para se instituir a terapia hormonal na menopausa (PARDINI, 2014).

\section{Tromboembolismo venoso}

O risco de trombose venosa está aumentado próximo de duas vezes mais no uso de terapia hormonal, principalmente se associado a fatores relativos à paciente como obesidade, idade maior que 60 anos, trombofilia, e a fatores referentesà dosagem, via de administração e associação do estrogênio com progestágeno (PARDINI, 2014).

\section{Doenças cardiovasculares}

A pressão sistólica eleva-se com a idade, sendo maior o aumento na mulher após a menopausa, predispondo-a cerca de duas vezes mais, ao risco relativo de isquemia cardíaca com risco de infarto segundo estudos epidemiológicos. É calorosa a discussão atual acerca das prevenções primária e secundária da DCV com a reposição estrogênica e/ou estroprogestogênica. Estudos científicos experimentais são claros em demonstrar o efeito benéfico dos estrogênios sobre os vasos sanguíneos, reduzindo o risco de DCV em aproximadamente 50\% (LOMÔNACOet al, 2015). 
Os efeitos negativos da TH em relação a DCV têm sido relacionados ao início da terapia com mulheres com idade avançada, uso de doses altas de estrógeno, via de administração e intervalo de tempo após a menopausa. De fato, há evidências de que, quando o TH é iniciado precocemente (até 10 anos após a menopausa ou após a histerectomia), ele pode realmente diminuir o risco cardiovascular. (PARDINI, 2014).

\section{Câncer de mama}

Segundo Giacomini (2006) o hipoestrogenismo leva a hipotrofia das mamas com diminuição de tamanho, tornando-as flácidas, havendo substituição gradual do parênquima por gordura. Existem evidências controvérsias da relação da TH com o câncer de mama, no entanto, pode-se afirmar que a percentagem de densidade mamária (PMD) é aumentada com o uso de estrógeno isolado ou associado à progesterona, sendo a PMD um forte indicador de risco de câncer de mama.

De acordo com a SOBRAC (2014) a terapia hormonal está contraindicada em mulheres que tiveram câncer de mama e nos casos em que há lesões de alto risco para câncer de mama futuro, como em hiperplasias com atipias, carcinoma in situ, mutações BRCA-1, BRCA-2 ou antecedentes familiares significativos, não havendo estudos suficientes que afirmem aumento dos riscos para câncer de mama, além dos que já apresentam.

\section{Câncer de Endométrio}

A hiperplasia do endométrio pode ocorrer sob o estímulo estrogênico. Na terapia para mulheres com útero, utiliza-se estrogênio associado à progesterona, visto que a principal e essencial atividade exercida pelo progestagénio é a transformação do endométrio de sua forma proliferativa em secretora e, portanto, proteção endometrial contra hiperplasia e câncer (MONTERROSA-CASTRO et al 2014).

A dose diária ideal de progestogênio deve inibir a atividade mitótica dentro de um curto período ( $\leq 2$ mitoses / 1.000 células glandulares ao final de um único tratamento) e não deve induzir hiperplasia endometrial (MANSON, 2013).

Além da dose, um fator significativo é também a duração do uso (pelo menos 10 dias no tratamento cíclico e 12 dias na terapia sequencial). A melhor proteção endometrial é obtida com o regime terapêutico contínuo (WHITMERet al, 2011).

\section{Síndrome Climatérica}

Muitos estudos de coorte e estudos transversais foram realizados com o objetivo de caracterizar sintomas climatéricos. Os mais consistentemente encontrados foram ondas de calor e secura vaginal, que juntamente com uma variedade de outros sintomas, frequentemente são designados como síndrome do climatério (MONTERROSA-CASTROet al, 2016).

\section{Sintomas vasomotores}

A duração das ondas de calor (fogachos) em relação ao início da menopausa foi objeto de um estudo de coorte recentemente publicado com 436 mulheres na pré-menopausa com idades entre 35 e 47 anos, das quais 90 desenvolveram ondas de calor leves, enquanto 259 desenvolveram ondas de calor moderadas a graves e 55 não tinha nenhum (WHITMERet al, 2011).

Em usuárias de hormonioterapia, houve uma redução de $75 \%$ na frequência e $87 \%$ na severidade dos sintomas vasomotores, segundo uma revisão sistemática do Instituto Cochrane (PARDINI, 2014).

Muitos estudos duplo-cegos, controlados por placebo, também demonstraram que a TH alivia os sintomas vasomotores. Os efeitos colaterais relatados incluem sensibilidade mamária, sangramento uterino, hemorragia, artralgia, alterações emocionais (irritabilidade, perda de motivação, depressão, outros) e, menos comumente, náuseas, vômitos, cefaleia, alterações de peso, erupção cutânea e prurido (risco relativo 1,41; intervalo de confiança de 95\%, 1,00-1,99) (PARDINI, 2014).

REAS/EJCH | Vol. 11 (4) | e244 | DOI: https://doi.org/10.25248/reas.e244.2019 Página 4 de 7 


\section{Sintomas urogenitais}

$\mathrm{Na}$ fase climatérica o hipoestrogenismo leva a diminuição da elasticidade do epitélio vaginal, evoluindo com atrofia e perda da rugosidade e lubrificação escassa com estímulo sexual, estando geralmente associado a ocorrência de prurido e irritação locais. É comum também manifestações como a incontinência urinária (IU) (afetando 10 a 30\% das mulheres entre 50 e 64 anos), bexiga hiperativa (BHA) e infecções recorrentes do trato urinário (IRTU) (ORCESI, et al., 2003).

Os estrogênios têm efeitos proliferativos diretos no epitélio uretral e vesical. Outros efeitos incluem acúmulo do epitélio vaginal e reconstituição da flora vaginal, resultando em menor frequência de colpite. Em ensaios de pequena escala, a ET vaginal reduziu significativamente a frequência de infecções do trato urinário. $O$ estrogênio vaginal é recomendado para o tratamento de infecções recorrentes do trato urinário tanto pela North American Menopause Society quanto pela diretriz Alemã. O risco relativo é reduzido em $36 \%$ para 75\% (PARDINI, 2014).

\section{Alterações Ósseas}

Verificou-se que a TH reduz a incidência de fraturas em estudos observacionais e em ensaios clínicos controlados e randomizados. A taxa de fratura clínica é reduzida, assim como as chamadas fraturas associadas à osteoporose. Embora o a Terapia estrogênica-progesterona seja um meio eficaz de prevenir a osteoporose, ela não pode ser recomendado como terapia de primeira linha. Após a suspensão da terapia hormonal, observa-se a interrupção do seu efeito protetor (SANTEN et al., 2010).

\section{Alterações do humor e da cognição}

Existem indicadores de que alterações nas funções cognitivas, concentração e memória sejam também afetadas pela falta do estrogênio. Estima-se que 33\% das mulheres sofrerão pelo menos um episódio de depressão maior durante a vida, com prevalência de $9 \%$ no climatério. No entanto, existem discordâncias acerca de haver aumento da incidência de depressão nesta fase (FREEMAN, 2011).

Receptores de estrogênios foram encontrados em áreas do cérebro envolvidas na cognição (córtex, lobos temporais e sistema límbico), podendo-se relacionar as dificuldades na memória recente e da concentração em mulheres na perimenopausa no hipoestrogenismo, tendo-se evidenciado melhora na memória de curto e longo prazo após a terapia hormonal (GIACOMINI, 2006).

No caso de iniciar a terapia estrogênica, em mulheres com menos de 60 anos, pode haver diminuição do risco de doença de Alzheimer (PARDINI, 2014).

\section{Câncer colorretal}

Foi identificado uma diminuição da incidência de câncer colorretal em estudos observacionais na terapia hormonal tempo-dependente, não devendo ser na prevenção da doença, mas podendo ser considerada nos casos de risco aumentado (GIACOMINI, 2006).

\section{Diabetes}

No climatério há aumento do risco de desenvolver diabetes melito tipo II (DMII), principalmente na existência de obesidade central e sedentarismo, podendo esse risco ser diminuído com o uso da terapia de estrogênio isolado ou associado à progesterona (PARDINI, 2014).

\section{Principais cuidados e processos na administração de terapia hormonal no climatério Diagnóstico}

Além do parâmetro idade cronológica de 40 e 65 anos, o climatério pode ser identificado pelos sintomas (critérios clínicos) e exames laboratoriais (critérios hormonais). A menopausa tem sido diagnosticada retrospectivamente por amenorréia no período de pelo menos um ano, após os 40 anos, associada a baixas concentrações de estrogênios (estradiol) no sangue, aumento dos níveis séricos de hormônio folículo- 
estimulante (FSH), acima de $40 \mathrm{Ul} / \mathrm{ml}$, e/ou baseada em estudos de estímulos, como o teste de supressão com progesterona (GIACOMINI; MELLA, 2006).

No entanto a dosagem de hormônios não é absolutamente necessária para o diagnóstico de climatério, não sedo rotineira diante do quadro clínico. Porém, em alguns casos como pacientes que ainda menstruam, apresentando sintomas vasomotores e irritabilidade sem estar relacionada a outras causas, a dosagem apenas do FSH pode contribuir com o diagnóstico (SANCHES et al., 2010).

Outro teste que pode ser válido no diagnóstico de mulheres com amnorréia é o teste de progesterona, que indicam dosagens muito baixas de estrogênio, quando o resultado é negativo (GIACOMINI; MELLA, 2006).

Pode-se lançar mão de outros exames complementares para se definir se a mesma provém da falência ovariana ou outras causas como tumores hipotalâmicos, hipotireodismo e etc. Destacando-se os exames de imagem como: mamografia, ultra-sonografia pélvica, e densitometria óssea; exames laboratoriais: hemograma, glicemia, fosfatase alcalina, lipidograma, cálcio e fósforo séricos, calciúria de 24; teste da progesterona e demais exames como a colposcopia e colpocitologia oncológica. Sendo essencial realizar ao menos a mamografia, a citologia oncótica e o teste provocativo de progestagênios na impossibilidade dos demais (GIACOMINI; MELLA, 2006).

\section{Pontos fundamentais quanto à indicação da terapia hormonal no climatério}

Antes que qualquer terapia hormonal seja iniciada, a indicação deve ser cuidadosamente considerada; 0 paciente deve ser avaliado com extensa anamnese e exame físico, incluindo um exame ginecológico. Só assim o médico pode detectar certas contraindicações e riscos para a saúde que possam surgir se a TH for iniciada (WHITMER et al, 2011).

Quanto a duração da TRH, aconselha-se que não seja a longo prazo. Conforme a Food and DrugAdministration, a North American Menopause Society e a Organização Panamericana de Saúde (OPAS) a TRH deve se ministrada na forma de doses mais baixas e em períodos curtos, até a ausência de sintomas, não devendo exceder o período de 5 anos (WHITMER et al, 2011).

\section{Contraindicações}

Segundo a SOBRAC (2014) as contraindicações da terapia hormonal são relativas à análise individualizada de cada caso, podendo, mesmo em situações de contraindicação, ser usada como alternativa de acordo com o juízo clínico e as singularidades de cada caso. Devendo-se atentar para a escolha da melhor via de administração, dose e regime terapêutico a serem empregados, bem como o tempo de tratamento e a decisão conjunta com a paciente. As contraindicações são: Doença hepática descompensada • Câncer de mama • Câncer de endométrio • Lesão precursora para câncer de mama Porfiria • Sangramento vaginal de causa desconhecida - Doenças coronariana e cerebrovascular - Doença trombótica ou tromboembólica venosa $\bullet$ Lúpus eritematoso sistêmico $•$ Meningeoma.

\section{Vias de administração}

Em relação às vias de administração temos: a via oral que precisa de doses hormonais aumentadas, devido o seu efeito de primeira passagem pelo fígado e metabolização na parede intestinal, porém por ser uma via de baixo custo e de possível reajuste ou interrupção na dose, torna-se uma via de uso prático, além de produzir maior efeito no aumento do HDL, diminuição do LDL, aumento nos triglicérideos, e estimula a produção de angiotensinogênio que pode elevar a pressão arterial, e de proteínas transportadoras de hormônios sexuais. A via transdérmica (sob a forma de gel e adesivos), que possui uma absorção elevada, não estando sujeita à inativação hepática, precisando assim baixas doses. A via vaginal que mais utilizada no tratamento de sintomas vulvovaginais ou urinários. Além das demais vias como a intramuscular, parenteral, intranasal, intrauterina (DIU) e os implantes subcutâneos (GIACOMINI, 2006). 
Tudo deixa claro que a TH pode ser um método eficaz para tratar sintomas climatéricos. A decisão de dar ou não a TH oral ou parenteral depende em grande parte do estado de saúde do paciente individual. A meticulosa anamnese é necessária em qualquer caso antes do início do tratamento. Os esteróides sexuais podem afetar o risco de desenvolver certas doenças. Uma compreensão completa desses riscos é importante, pois eles não dependem apenas do perfil de saúde do paciente, mas também do regime hormonal específico usado (ET, EPT) e da duração da administração. Todos os pacientes devem ser adequadamente informados sobre os riscos do tratamento (WENDER;et al 2014).

Epidemiologicamente pensando, estes riscos não deveriam limitar o uso da terapia hormonal. O papel do médico é essencial: assegurar os benefícios e prevenir os riscos. E isto é possível (PARDINI, 2014).

\section{CONCLUSÃO}

Os benefícios e riscos gerais da TH, incluíram alívio de ondas de calor e sintomas de atrofia urogenital e prevenção de fraturas e diabetes. Os riscos incluíram episódios venotrombóticos, acidente vascular cerebral e câncer de mama.Mulheres que iniciam a TH entre 50 e 59 anos ou menos de 10 anos após o início da menopausa, as tendências congruentes sugeriram benefício adicional. Os consensos atuais são concordantes quando expõem que os benefícios da TH são maiores ou absolutos quando estabelecida na perimenopausa. A mulher na faixa de 50 a 59 anos ou com tempo de menopausa menor que 10 anos é o grupo prioritário e se enquadra na faixa de mulheres onde os benefícios na maioria das vezes superam os riscos.

\section{REFERÊNCIAS}

1. BISOGNIN, Priscila. Costurando saberes e práticas de cuidado no climatério. Disponível em:http:/coral.ufsm.br/ppgenf/images/Dissertacoes_alunos/Dissertacao_Priscila_Bisognin.pdf.

2. FONSECA, J.J.S. Metodologia da pesquisa científica. Fortaleza: UEC, 2012.

3. FREEMAN EW, et al.Duration of menopausal hot flushes and associated risk factors. Obstetrics and gynecology, 2011;117:1095-1104.

4. GIACOMINI DR, MELLA EAC. Reposição Hormonal: vantagens e desvantagens. Semina: Ciências Biológicas e Saúde, Londrina, v. 27, n. 1, p.71-92, jan./jun. 2006.

5. LOMÔNACO C, et al. O impacto da menopausa nas relações e nos papéis sociais estabelecidos na família e no trabalho. Reprodclim. v. 30, n. 2, p. 58-66, 2015.

6. MANSON JE. Menopausal hormone therapy and health outcomes during the intervention and extended poststopping phases of the Women's Health Initiative randomized trials. JAMA, 2013 Oct;310(13):1353-68.

7. MONTERROSA-CASTRO, et al. Disfunción sexual em mujeres climatéricas afrodescendientes del Caribe Colombiano. latreia, v. 27, n. 1, p. $31-41,2014$.

8. PARDINI, Dolores. Terapia de reposição hormonal na menopausa. Arq Bras Endocrinol Metab, $2014 ; 5$.

9. RIBEIRO B, et al. Disfunção sexual feminina em idade reprodutiva: prevalência e fatores associados. Rev Port Med Geral Fam, 2013;29(01):16-24.

10. SANCHES TR, et al. Avaliação dos sintomas climatéricos na mulher em menopausa e pós menopausa em uso de proteína isolada de soja. J Health Sci Inst, v. 28, n. 2, p. 169-73, 2010.

11. SANTEN RJ, et al. Postmenopausal Hormone Therapy. J Clin Endocrinol Metab, July 2010, 95(Suppl 1):S1-S66.

12. SOBRAC (2014). Associação Brasileira de climatério. Disponível em http://sobrac.org.br/index.php acessado dia 10 de janeiro de 2018.

13. VALENÇA CN, GERMANO RM. Concepções de mulheres sobre menopausa e climatério. Rev. Rene, Fortaleza, v. 11, n. 1, 2010.

14. WENDER, et al. Consenso Brasileiro de Terapêutica Hormonal da Menopausa - Associação Brasileira de Climatério (SOBRAC) - São Paulo: Leitura Médica, 2014.

15. WHITMER RA, et al. Timing of hormone therapy and dementia: the critical window theory revisited. Ann Neurol, 2011;69:163169. 